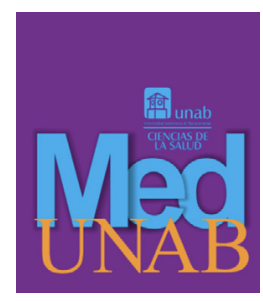

REVISTA DE LA FACULTAD

DE CIENCIAS DE LA SALUD

\title{
Enterocolitis necrotizante: hallazgos sociodemográficos, clínicos e histopatológicos en una serie de autopsias neonatales
}

Necrotizing entercolitis: socio-demographic, clinical and histopathological findings in a series of neonatal autopsies

Enterocolite necrosante: achados sociodemográficos, clínicos e histopatológicos em uma série de autópsias neonatais

Diana K. Sandoval-Martínez, MD., Esp. ${ }^{1}$ (D) , Mayra Z. Jaimes-Sanabria, MD. ${ }^{2}$ (D), Fanny L. Jiménez-Vargas, MD. ${ }^{2}$ (D) , Diego F. Chaparro-Zaraza, Est. ${ }^{3}$ (D), Edgar F. Manrique-Hernández, MD. ${ }^{2}$ (D) .

1. Médica, Especialista en Patología. Hospital Universitario de Santander, Departamento de Patología. Universidad Industrial de Santander, Bucaramanga, Santander, Colombia.

2. Médico y cirujano. Universidad Industrial de Santander, Bucaramanga, Santander, Colombia.

3. Estudiante de Medicina. Universidad Industrial de Santander, Bucaramanga, Santander, Colombia.

Correspondencia. Diana K. Sandoval. Facultad de Salud, Departamento de Patología, Universidad Industrial de Santander. Carrera 32 \#29-31. Bucaramanga, Santander, Colombia. Email.kt_sandoval@hotmail.com

\section{INFORMACIÓN DEL ARTÍCULO:}

Artículo recibido: 13 de agosto de 2019

Artículo aceptado: 20 de enero de 2020

DOI: https://doi.org/10.29375/01237047.3682

Cómo citar. Sandoval-Martínez DK, Jaimes-Sanabria MZ, Jiménez-Vargas FL, Chaparro-Zaraza DF, ManriqueHernández EF. Enterocolitis necrotizante: hallazgos sociodemográficos, clínicos e histopatológicos en una serie de autopsias neonatales. MedUNAB. 2020;23(1):35-42. Doi: 10.29375/01237047.3682

\section{RESUMEN}

Introducción. La enterocolitis necrotizante es un trastorno caracterizado por la necrosis isquémica de la mucosa intestinal, es la enfermedad gastrointestinal más grave que afecta a los neonatos, con alta morbilidad y mortalidad, principalmente en prematuros. El objetivo del presente estudio es describir las características clínicas y anatomopatológicas de los recién nacidos fallecidos con enterocolitis necrotizante, 
diagnosticados en un hospital de alta complejidad. Metodología. Este es un estudio descriptivo retrospectivo de 21 casos de autopsias médico-científicas hechas en un hospital de alta complejidad del nororiente colombiano, con hallazgos anatomopatológicos de enterocolitis necrotizante, realizadas entre enero de 2013 y julio de 2017. Resultados. El 85.7\% de los recién nacidos eran pretérminos, un igual porcentaje presentaba un peso menor a 2,500 gramos al nacer. Respecto a los antecedentes maternos el $14.3 \%$ tuvieron espectro de trastornos hipertensivos asociados al embarazo y el $23.8 \%$ infección materna. Los tres sitios más frecuentes de ubicación de enterocolitis necrotizantes fue íleon, colon ascendente y colon transverso. Discusión. Según algunos autores, hasta el 85\% de todos los casos de enterocolitis necrotizante ocurren en pacientes prematuros, especialmente en bebés con peso extremadamente bajo al nacer. Hay formas de enterocolitis necrotizante que ocurren en bebés a término y, generalmente, están asociadas con factores predisponentes, resultados compatibles con lo que encontramos en esta investigación. Conclusiones. Este estudio elaborado con población colombiana se correlaciona con lo descrito en la población mundial en la cual la enterocolitis necrotizante se presenta más en los recién nacidos pretérmino y con bajo peso al nacer.

Palabras clave:

Enterocolitis Necrotizante; Recién Nacido; Recién Nacido Prematuro; Recién Nacido de Bajo Peso; Autopsia.

\begin{abstract}
Introduction. Necrotizing entercolitis is a disorder characterized by the ischemic necrosis of intestinal mucosa. It is the most serious gastrointestinal disease affecting neonates, with high morbidity and mortality rates, mainly among premature newborns. The purpose of this study is to describe the clinical and anatamo-pathological characteristics of deceased newborns with necrotizing entercolitis, diagnosed at a high-complexity hospital. Methodology. This is a descriptive retrospective study of 21 cases of medical-scientific autopsies performed at a high-complexity hospital in northeastern Colombia, with anatomo-pathological findings of necrotizing entercolitis, performed between January 2013 and July 2017. Results. $85.7 \%$ of the newborns were pre-term, and the same percentage had weight at birth below 2.5 kilograms. Regarding the mothers' background, $14.3 \%$ displayed a spectrum of hypertensive disorders associated with pregnancy, and 23.8\% had maternal infections. The three most frequent locations of necrotizing entercolitis were the ileum, ascending colon and transverse colon. Discussion. According to some authors, up to $85 \%$ of all cases of necrotizing entercolitis occur in premature patients, especially in babies with extremely low weight at birth. There are forms of necrotizing entercolitis that occur in full term babies, and they are generally associated with contributing factors, which is consistent with the findings of this study. Conclusions. This study of a Colombian population is consistent with other descriptions of the global population, where necrotizing entercolitis arises more frequently in pre-term newborns and low weight at birth.
\end{abstract}

Keywords:

Necrotizing Entercolitis; Newborn; Premature Infant; Low Birth Weight Infant; Autopsy.

\title{
RESUMO
}

Introdução. A enterocolite necrosante é um transtorno caracterizado por necrose isquêmica da mucosa intestinal é a doença gastrointestinal mais grave que afeta aos recém-nascidos, com alta morbimortalidade, principalmente em prematuros. O objetivo deste estudo é descrever as características clínicas e anatomopatológicas dos recém-nascidos que morreram com enterocolite necrosante, diagnosticados em um hospital de alta complexidade. Métodos. Este é um estudo descritivo retrospectivo de 21 casos de autópsias médico-científicas feitas em um hospital de alta complexidade no nordeste da Colômbia, com achados anatomopatológicos de enterocolite necrosante, realizadas entre janeiro de 2013 e julho de 2017. Resultados. $85.7 \%$ dos recém-nascidos eram prematuros. Uma porcentagem igual tinha um peso menor que 2,500 gramas ao nascer. Em relação à história materna, 14.3\% possuíam espectro de transtornos hipertensivos associados à gravidez e 23.8\% à infecção materna. Os três locais mais frequentes de enterocolite necrosantes foram íleo, colo ascendente e colo transverso. Discussão. Segundo alguns autores, até $85 \%$ de todos os casos de enterocolite necrosante ocorrem em prematuros, 
principalmente em bebês com peso extremamente baixo ao nascer. Existem formas de enterocolite necrosante que ocorrem em bebês a termo e, geralmente, estão associadas a fatores predisponentes, resultados compatíveis com os achados nesta pesquisa. Conclusão. Este estudo realizado com população colombiana está correlacionado com o descrito na população mundial em que a enterocolite necrosante ocorre mais nos recém-nascidos prematuros e com baixo peso ao nascer.

Palavras-chave:

Enterocolite Necrosante; Recém-Nascido; Recém-Nascido Prematuro; Recém-Nascido de Baixo Peso; Autópsia.

\section{Introducción}

La enterocolitis necrotizante (ECN) es un trastorno caracterizado por necrosis isquémica de la mucosa intestinal, inflamación, invasión de organismos formadores de gas y difusión de gas en los sistemas muscular y venoso portal. Lo anterior la convierte en la enfermedad gastrointestinal más grave en los recién nacidos $(\mathrm{RN})(1)$. Se asocia con una alta morbimortalidad (2). Su diagnóstico clínico es un reto, pero en general se basa en la presencia de intolerancia a la alimentación, distensión abdominal, heces con sangre y el hallazgo en imágenes abdominales de gas intramural (neumatosis intestinal), neumoperitoneo o gas hepatobiliar. Sin embargo, el diagnóstico definitivo de ECN se realiza a partir de muestras quirúrgicas intestinales o post mortem con hallazgos histológicos de inflamación intestinal, infarto y necrosis (3).

La incidencia global de ECN es cercana a uno en 1,000 nacidos vivos (4); asimismo, la incidencia en América Latina y en Colombia es incierta debido a la escasez de datos confiables relacionados con inconsistencias en el diagnóstico y la recopilación de datos (5). Presentándose hasta en el 7\% de los RN en cuidados intensivos neonatales (6); con tasa de mortalidad aproximada del $20 \%$ al 30\% $(7,8)$. Se observa comúnmente en RN prematuros, pero alrededor del $13 \%$ de los casos se presentan en bebés a término (1) y afecta del 3\% al 5\% de todos los $\mathrm{RN}$ de bajo peso al nacer $(6,9)$.

La ECN es una enfermedad multifactorial con una patogenia poco conocida y controversial; sin embargo, hay dos posibles mecanismos implicados en la fisiopatología: en la primera se considera que el flujo sanguíneo alterado en los vasos mesentéricos conduce a isquemia secundaria del intestino, y la segunda propone un mecanismo mediado por respuesta inflamatoria (10). Más del 90\% de los casos se presentan en recién nacidos de bajo peso al nacer $(<1,500$ gramos $)$ y en menores de 32 semanas de gestación (11).
La ECN representa una prioridad clínica y de investigación, con un costo económico y psicosocial alto, por lo tanto, una mejor comprensión de las características de los pacientes con ECN podría llevar a plantear medidas preventivas en pacientes más vulnerables; de esta forma, el objetivo de este estudio es describir las características clínicas y anatomopatológicas de los RN fallecidos con ECN, diagnosticados en un hospital de alta complejidad del nororiente colombiano.

\section{Metodología}

Se realizó un estudio descriptivo retrospectivo que incluyó a $\mathrm{RN}$ a quienes se les practicó autopsia médico científica en un hospital de alta complejidad (Hospital Universitario de Santander), entre enero de 2013 y julio de 2017. En este periodo se realizaron 2,134 autopsias médico-científicas, de las cuales 334 correspondían a autopsias neonatales. Los criterios de inclusión fueron la confirmación diagnóstica basándose en criterios clínicos, radiológicos e histopatológicos de ECN, la presencia de información acerca de edad gestacional, peso al nacer, edad materna, información clínica materna y del neonato. Los criterios de exclusión fueron la ausencia de los criterios de inclusión. En total, 21 neonatos con ECN cumplieron con el criterio de inclusión.

Se tuvieron en cuenta las siguientes variables: características prenatales, perinatales y postnatales: edad materna, antecedentes maternos de importancia de tipo infecciosos (1), ruptura prematura de membranas (2), espectro de trastornos hipertensivos asociados al embarazo, paridad, embarazo gemelar, vía de parto (vaginal o cesárea) (8), edad gestacional al nacer (semanas), sexo (1), presencia de lesión hipóxico-isquémica (12), peso al nacer (gramos) (2), tiempo de vida extrauterina (días), manejo quirúrgico de la enterocolitis (9), comorbilidades como malformaciones cardíacas congénitas (6) y malformaciones gastrointestinales de los neonatos (13), ubicación de enterocolitis (6) y causa de muerte. 
La recolección de datos se realizó a través de los informes de autopsias médico científicas de los pacientes. Para la elaboración de la base de datos se utilizó el software Microsoft Excel® y para el análisis de datos se usó el software Stata, versión 13 (StataCorp LP, College Station, TX, USA). Se realizó extracción de frecuencias y porcentajes de las variables categóricas y medidas de tendencia central, con sus valores mínimos y máximo para el caso de las variables continuas.

Este estudio sigue las normas éticas elaboradas en la declaración de Helsinki modificada (Brasil 2013) $\mathrm{y}$ al reporte de Belmont. Adicionalmente, según la normatividad en salud pública del Instituto Nacional de Salud de Colombia, se debe realizar autopsias al 100\% de los casos de muerte perinatal y neonatal tardía donde la causa de la muerte no esté determinada (14); por lo tanto, no se requirió consentimiento informado.

\section{Resultados}

Durante el período de estudio se reclutaron 21 casos con ECN. En la tabla 1 se describen las características generales de la población.

El $14.3 \%(n=3)$ corresponden a madres adolescentes $(<18$ años) y no se presentó ningún caso de madre añosa (>38 años). El 85.7\% ( $\mathrm{n}=18)$ eran recién nacidos con peso menor a 2,500 gramos; $19.1 \%(n=4)$ con bajo peso al nacer $(<1,500$ gramos) y $23.8 \%(n=5)$ con peso extremadamente bajo al nacer $(<1,000$ gramos). (Tabla 2$)$

En los antecedentes maternos se encontró que 38.1\% $(\mathrm{n}=8)$ presentó al menos un antecedente de importancia y el $19.1 \%(n=4)$ presentó más de un antecedente materno. El 14.3\% $(\mathrm{n}=3)$ tuvieron espectro de trastornos hipertensivos asociados al embarazo; el 23.8\% $(n=5)$ infección materna, de estas un caso con sífilis gestacional tratada y el $33.3 \%(\mathrm{n}=7)$ presentó ruptura prematura de membranas.

Respecto a los factores de riesgo neonatales, el 38.1\% $(\mathrm{n}=8)$ presentó al menos una patología asociada y el $33.3 \%$ presentó más de una. En el $52.4 \%(\mathrm{n}=11)$ de los casos se diagnosticó lesión hipóxico isquémica (asfixia perinatal). El 14.3\% $(\mathrm{n}=3)$ presentó restricción del crecimiento intrauterino. El $14.3 \%(\mathrm{n}=3)$ presentaron kernicterus neonatal, además, se detectaron malformaciones cardiacas en el $28.6 \%(n=6)$, en pared abdominal de tipo gastrosquisis un $9.5 \%(n=2)$ y gastrointestinales (enfermedad de Hirschsprung y atresia del intestino delgado tipo I con dilatación quística proximal) en un $4.6 \%(n=1)$, así mismo, un caso de trisomía 13 , un caso
Tabla 1. Características de la población

\begin{tabular}{|c|c|}
\hline \multicolumn{2}{|l|}{ Sexo } \\
\hline Hombre & $11(52.4 \%)$ \\
\hline Mujer & $10(47.6 \%)$ \\
\hline \multicolumn{2}{|l|}{ Edad materna (años) } \\
\hline Media & $24,95 \pm 6.58(15-38)$ \\
\hline \multicolumn{2}{|l|}{ Paridad } \\
\hline Primigestante & $7(33.3 \%)$ \\
\hline Multigestante & $11(52.4 \%)$ \\
\hline Sin dato & $2(14.3 \%)$ \\
\hline \multicolumn{2}{|l|}{$\begin{array}{l}\text { Edad gestacional } \\
\text { (semanas) }\end{array}$} \\
\hline Media & $31.2(24-40)$ \\
\hline $\begin{array}{r}\text { Recién nacidos } \\
\text { término }\end{array}$ & $3(14.3 \%)$ \\
\hline Recién pretérmino & $18(85.7 \%)$ \\
\hline \multicolumn{2}{|l|}{ Embarazo gemelar } \\
\hline $\mathrm{Si}$ & $3(14.3 \%)$ \\
\hline No & $18(85.7 \%)$ \\
\hline \multicolumn{2}{|l|}{ Vía parto } \\
\hline Cesárea & $12(57.1 \%)$ \\
\hline Vaginal & $9(42.9 \%)$ \\
\hline \multicolumn{2}{|l|}{$\begin{array}{l}\text { Peso al nacer } \\
\text { (gramos) }\end{array}$} \\
\hline Media & $1609 \pm 699(490-3000)$ \\
\hline \multicolumn{2}{|l|}{$\begin{array}{l}\text { Tiempo de vida } \\
\text { extrauterina (días) }\end{array}$} \\
\hline Media & $11 \pm 8(2-27)$ \\
\hline
\end{tabular}

Fuente: Elaborada por autores.

de síndrome de crigler-Najjar y uno de síndrome disorganization-like. El 28.6\% $(\mathrm{n}=6)$ recibieron tratamiento quirúrgico.

En el $62 \%(n=13)$ se consideró que la ECN fue la causa básica de muerte, en los casos restantes la ECN fue un hallazgo adicional y la causa básica de muerte fue hemorragia intraventricular $(n=3)$, hemorragia intraalveolar $(\mathrm{n}=1)$, gastrosquisis $(\mathrm{n}=2)$ y dos casos por anomalías congénitas de tipo comunicación interventricular y síndrome disorganization-like.

La frecuencia de localización de ECN fue íleon 89.5\% $(n=17)$, colon ascendente $47.4 \%(n=9)$, colon transverso $31.6 \%(n=6)$, yeyuno $26.3 \%(n=5)$, ciego $26.3 \%$ $(\mathrm{n}=5)$, colon descendente $21.1 \%(\mathrm{n}=4)$, válvula ileocecal $10.5 \%(\mathrm{n}=2)$ y duodeno $5.3 \%(\mathrm{n}=1)$. (Tabla 2$)$ (figura 1). 
Tabla 2. Características clínicas neonatales de 21 casos de enterocolitis necrotizante

\begin{tabular}{|c|c|c|c|}
\hline Caso & EG (sem) & Peso (g) & $\begin{array}{l}\text { Ubicación anatómica } \\
\text { enterocolitis }\end{array}$ \\
\hline 1 & 36.2 & 1590 & Íleon, CA, CT, CD \\
\hline 2 & 31.4 & 1540 & Ciego \\
\hline 3 & 28.3 & 1040 & Íleon, CT \\
\hline 4 & 31.5 & 1830 & Yeyuno, íleon, CA \\
\hline 5 & 31 & 1810 & $\begin{array}{l}\text { Íleon, Ciego, CA, CT, } \\
\text { CD }\end{array}$ \\
\hline 6 & 33 & 1790 & $* * * *$ \\
\hline 7 & 27.6 & 900 & Yeyuno, íleon \\
\hline 8 & 38.5 & 3000 & Íleon \\
\hline 9 & 30 & 1450 & Íleon, VIC, CA, CT \\
\hline 10 & 28 & 780 & Yeyuno, íleon \\
\hline 11 & 25.4 & 990 & Íleon \\
\hline 12 & 30.5 & 1840 & Íleon \\
\hline 13 & 28 & 940 & Íleon \\
\hline 14 & 33.3 & 1510 & Íleon, ciego, CA \\
\hline 15 & 37 & 3000 & Íleon \\
\hline 16 & 33 & 2190 & $* * * *$ \\
\hline 17 & 33 & 2200 & $\begin{array}{l}\text { Duodeno, íleon, Yeyuno, } \\
\text { VIC, CA, CT, CD }\end{array}$ \\
\hline 18 & 24 & 490 & Íleon, ciego \\
\hline 19 & 27.6 & 1080 & Yeyuno, íleon, CA \\
\hline 20 & 28 & 1220 & Íleon, CA \\
\hline 21 & 40 & 2600 & $\mathrm{CA}, \mathrm{CT}, \mathrm{CD}$ \\
\hline
\end{tabular}

EG: Edad Gestacional, CA: colon Ascendente, CT: Trasverso, CD: descendiente, VIC: Válvula Ileocecal. ****: No se reporta dato de localización.

Fuente: Elaborada por autores.

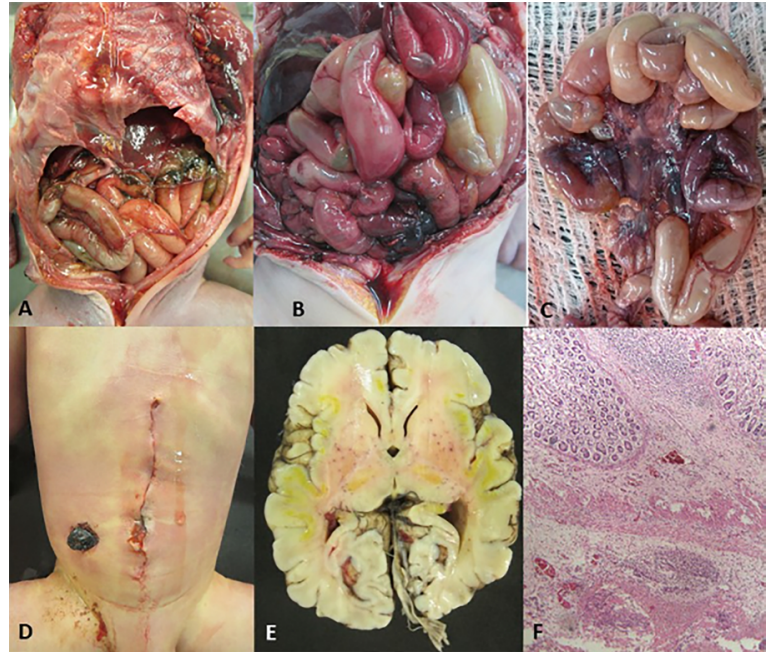

Figura 1. Letra A, Caso 5 compromiso de colon con peritonitis y membranas fibrinosas; Letra $\mathrm{B}$, Caso 7 compromiso de yeyuno e íleon con neumatosis evidente; Letra $\mathrm{C}$, Caso 9 compromiso de íleon, válvula ileocecal, colon ascendente y transverso; Letra D, Caso 1 paciente con ictericia e ileostomía; Letra E, caso 12 Caso con kernicterus asociado; Letra F, caso 12, corte histológico del íleon en la que se reconoce inflamación trasmural con daño de las fibras musculares

Fuente: Elaborada por autores.

\section{Discusión}

En prematuros con ECN se ha descrito que la inmadurez intestinal genera susceptibilidad debido a la disminución de la protección y reparación de la pared intestinal $(6,15)$. Adicionalmente, el bajo peso al nacer es un factor de riesgo para el desarrollo y pronóstico de la ECN. Estudios refieren que existe una asociación inversa entre la mortalidad y el peso al nacer $(2,6)$, y según algunos autores hasta el $85 \%$ de todos los casos de ECN se presentan en pacientes prematuros principalmente, en los $\mathrm{RN}$ de peso extremadamente bajo (4). Resultados compatibles con lo encontrado en este estudio, en el cual el $85.7 \%$ son nacidos pretérminos y el mismo porcentaje presentaron pesos inferiores a 2,500 gramos.

Existen formas de ECN que se presentan en los bebés a término y suelen estar asociados a factores predisponentes como restricción del crecimiento intrauterino, asfixia perinatal (16), ruptura prolongada de membranas (17), insuficiencia respiratoria, sepsis, convulsiones neonatales, hipoglucemia, hipotermia e hipertermia (13), transfusiones sanguíneas, defectos del tubo neural (17), gastrosquisis (13), y cardiopatía congénita (16). En el presente estudio el 14.3\% $(n=3)$ de los casos se trataban de RN a término, en los que se 
encontró dos casos con lesión hipóxico-isquémica y un caso con malformación cardiaca de tipo comunicación interventricular; los casos de lesión hipóxico isquémica se asociaron de manera independiente a ruptura prematura de membranas y restricción del crecimiento intrauterino.

Varias investigaciones detectaron una mayor frecuencia de ECN en neonatos prematuros con cardiopatía congénita compleja grave (18); quienes presentan un riesgo mayor de desarrollar ECN, debido a la disminución de la perfusión mesentérica $(17,19)$. Datos concordantes con esta investigación donde el 83.3\% $(n=5 / 6)$ de los casos con ECN y cardiopatía congénita eran RN prematuros.

El concepto de disbiosis es un tema relevante en la patogénesis de la ECN. La disbiosis se refiere al desequilibrio entre las bacterias protectoras $\mathrm{y}$ las bacterias oportunistas perjudiciales (20). Los RN prematuros presentan una pobre colonización bacteriana protectora, por su inmadurez (21). Otros factores causantes de una menor exposición a las bacterias protectoras son el parto por cesárea, la alimentación con fórmula, la terapia antibacteriana empírica temprana y la terapia con bloqueadores de histamina (20). En esta investigación, más de la mitad de los casos la vía del parto fue parto por cesárea, lo cual pudo haber sido un factor contribuyente en la presentación de ECN.

La afectación por ECN según la edad gestacional es colón en recién nacidos término e intestino delgado en los recién nacidos pretérmino; siendo el yeyuno el lugar más frecuentemente afectado en los bebés extremadamente prematuros $(<30$ semanas $)$ e íleon en los bebés prematuros (30-36 semanas) (6). Datos discordantes con este estudio. Se sugiere para futuros estudios ampliar la muestra, para confirmar o refutar los hallazgos.

E1 25\% de los neonatos con ECN se someten a un manejo quirúrgico, la mortalidad postoperatoria se encuentra entre el 20 al $60 \%$. Las complicaciones perioperatorias se presentan hasta en el $70 \%$ de los casos e incluyen muerte, complicaciones relacionadas con enterostomía, estenosis intestinales, sepsis, peritonitis e infecciones de heridas (9). Adicionalmente, las consecuencias a largo plazo de ECN son estenosis intestinal, síndrome del intestino corto, deterioro en el crecimiento y problemas de desarrollo neurológico $(20,22)$.

La enterocolitis necrotizante genera un síndrome de respuesta inflamatoria sistémica, este se ha relacionado como potenciadores de la neurotoxicidad de la bilirrubina, siendo mayor en RN prematuros $(23,24)$. En esta muestra se diagnosticaron 3 casos de kernicterus neonatal, todos RN menores de 36.6 semanas. También existe una asociación entre el espectro de trastornos hipertensivos asociados al embarazo que genera hipoxia fetal y restricción del crecimiento intrauterino, que puede ser el mecanismo subyacente que predispone al neonato a ECN, siendo la preeclampsia un factor de riesgo en recién nacidos prematuros y en recién nacidos con un peso al nacer inferior a 1,500 gramos (25). En este estudio los casos de espectro de trastornos hipertensivos asociados al embarazo y ECN se presentaron en recién nacidos prematuros, dos de los tres casos tenían un peso al nacer menor a 1,500 gramos, el tercero presentó restricción del crecimiento intrauterino.

Este estudio tiene limitaciones, ya que se realizó en una sola región del país, contó con una cohorte retrospectiva, solo se tuvo acceso a los informes de autopsia, y se desconoce el total de la población que desarrolló enterocolitis. A pesar de estas limitaciones, el estudio describe las características de los pacientes que desarrollan ECN en un centro de atención de alta complejidad en el nororiente colombiano y de los centros de atención médica cercanos, ya que, según la legislación colombiana, todo caso de muerte neonatal debe ser sometido a una investigación para definir su causa de muerte encontrando que los resultados concuerdan con lo descrito en la literatura.

\section{Conclusión}

La patogenia y la presentación clínica de la enterocolitis no está completamente dilucidada, este estudio llevado a cabo con población colombiana se correlaciona con lo descrito en la población mundial, en la cual la enterocolitis necrotizante se presenta más en los recién nacidos pretérmino, bajo peso al nacer, lesión hipóxico isquémica, malformaciones cardiacas en prematuros, espectro de trastornos hipertensivos asociados al embarazo e infección materna.

\section{Agradecimientos}

Al departamento de patología de la Universidad Industrial de Santander.

\section{Conflicto de intereses}

Nada que declarar. 


\section{Fuentes de financiamiento}

No se recibió ningún tipo de financiamiento.

\section{Referencias}

1. Stafford IA, Rodrigue E, Berra A, Adams W, Heard AJ, Hagan JL, et al. The strong correlation between neonatal early-onset Group B Streptococcal disease and necrotizing enterocolitis. European Journal of Obstetrics and Gynecology and Reproductive Biology. 2018;223,93-97. doi: https://doi.org/10.1016/j. ejogrb.2018.02.024.

2. Samuels N, Van-De-Graaf RA, De-Jonge RCJ, Reiss IKM, Vermeulen MJ. Risk factors for necrotizing enterocolitis in neonates: a systematic review of prognostic studies. BMC Pediatrics. 2017;17(1):105. doi: https://doi.org/10.1186/s12887-017-0847-3.

3. Neu J, Walker WA. Necrotizing Enterocolitis. The New England Journal of Medicine. 2011;364:25564. doi: https://doi.org/10.1056/nejmra1005408.

4. Moschopoulos C, Kratimenos P, Koutroulis I, Shah BV, Mowes A, Bhandari V. Review Article The Neurodevelopmental Perspective of Surgical Necrotizing Enterocolitis: The Role of the Gut-Brain Axis. Mediators of Inflammation. 2018:1-8. doi: https://doi. org/10.1155/2018/7456857.

5. Zani A, Pierro A. Necrotizing enterocolitis: controversies and challenges. F1000Research. 2015. doi: https://doi.org/10.12688/f1000research.6888.1.

6. Feldens L, Souza JCK, Fraga JC. There is an association between disease location and gestational age at birth in newborns submitted to surgery due to necrotizing enterocolitis. Jornal de Pediatria. 2018;94(3):320-324. doi: https://doi.org/10.1016/j. jped.2017.06.010.

7. Aceti A, Beghetti I, Martini S, Faldella G, Corvaglia L. Review Article Oxidative Stress and Necrotizing Enterocolitis: Pathogenetic Mechanisms, Opportunities for Intervention, and Role of Human Milk. Oxidative Medicine and Cellular Longevity. 2018:1-7. doi: https://doi.org/10.1155/2018/7397659.

8. Yang CC, Tang PL, Liu PY, Huang WC, Chen YY, Wang $\mathrm{H}$, et al. Maternal pregnancy-induced hypertension increases subsequent neonatal necrotizing enterocolitis risk. Medicine. 2018;97(31): e11739. doi: https://doi.org/10.1097/MD.0000000000011739.

9. Mutanen A, Pierro A, Zani A. Perioperative Complications Following Surgery for Necrotizing Enterocolitis. European Journal of Pediatric Surgery. 2018;28(2):148151. doi: https://doi.org/10.1055/s-0038-1636943.

10. Szpecht D, Neumann-Klimasińska N, Błaszczyński M, Seremak-Mrozikiewicz A, Kurzawińska G, Cygan D, et al. Candidate gene analysis in pathogenesis of surgically and non-surgically treated necro- tizing enterocolitis in preterm infants. Molecular and Cellular Biochemistry. 2018;439(1-2):53-63. doi: https://doi.org/10.1007/s11010-017-3135-5.

11. Rees CM, Eaton S, Pierro A. National prospective surveillance study of necrotizing enterocolitis in neonatal intensive care units. Journal of Pediatric Surgery. 2010; 45(7): 1391-1397. doi: https://doi.org/10.1016/j.jpedsurg. 2009.12.002.

12. Lu Q, Cheng S, Zhou M, Yu J. Risk Factors for Necrotizing Enterocolitis in Neonates: A Retrospective Case-Control Study. Pediatrics \& Neonatology. 2017;58(2):165-170. doi: https://doi.org/10.1016/j. pedneo.2016.04.002.

13. Müller MJ, Paul T, Seeliger S. Necrotizing enterocolitis in premature infants and newborns. Journal of Neonatal-Perinatal Medicine. 2016;16;9(3):233-42. doi: https://doi.org/10.3233/NPM-16915130.

14. Equipo Maternidad Segura Subdirección de Prevención, Vigilancia y Control en Salud Pública Instituto Nacional de Salud. Mortalidad Perinatal y Neonatal Tardía. Protocolo de vigilancia en salud pública. 2017:1-31p

15. Denning TL, Bhatia AM, Kane AF, Patel RM, Denning PW. Pathogenesis of NEC: Role of the innate and adaptive immune response. Seminars in Perinatology. 2017; 41(1):15-28. doi: https://doi.or$\mathrm{g} / 10.1053 / \mathrm{j}$. semperi.2016.09.014.

16. Hackam D, Caplan M. Necrotizing enterocolitis: Pathophysiology from a historical context. Seminars in Pediatric Surgery.2018; 27(1):11-8 doi: https://doi. org/10.1053/j.sempedsurg.2017.11.003.

17. Lau PE, Cruz SM, Ocampo EC, Nuthakki S, Style $\mathrm{CC}$, Lee $\mathrm{TC}$, et al. Necrotizing enterocolitis in patients with congenital heart disease: A single center experience. Journal of Pediatric Surgery. 2018;53(5):914-917. doi: https://doi.org/10.1016/j. jpedsurg.2018.02.014.

18. Duci M, Fascetti F, Marta L, Elena E, Maria P, Cavicchiolo E, et al. Neonatal independent predictors of severe NEC. Pediatric Surgery International. 2018;34(6):663-669. doi: https://doi.org/10.1007/ s00383-018-4261-1.

19. Lopez NL, Gowda C, Backes CH, Nandi D, Rn HM, Fichtner S, et al. Differences in midterm outcomes in infants with hypoplastic left heart syndrome diagnosed with necrotizing enterocolitis: NPCQIC database analysis. Congenital Heart Disease. 2018;13(4):512-518. doi: https://doi.org/10.1111/ chd.12602.

20. Agnoni A, Lazaros-Amendola C. Necrotizing enterocolitis: Current concepts in practice. Journal of the American Academy of Physician Assistants. 2017;30(8):16-21. doi: https://doi.org/10.1097/01. JAA.0000521131.85173.f9. 
21. Caplan MS, Fanaroff A. Necrotizing: A historical perspective. Seminars in Perinatology. 2017;41(1):26. doi: https://doi.org/10.1053/j.semperi.2016.09.012.

22. Rich BS, Dolgin SE. Necrotizing Enterocolitis. Pediatrics in Review. 2017;38(12):552-559. doi: https:// doi.org/10.1542/pir.2017-0002.

23. Watchko JF. Bilirubin-Induced Neurotoxicity in the Preterm Neonate. Clinics in Perinatology.

2016;43(2):297-311.doi: https://doi.org/10.1016/j. clp.2016.01.007.

24. Watchko JF, Maisels MJ. The enigma of low bilirubin kernicterus in premature infants: why does it still occur, and is it preventable? Seminars in Perinatology. 2014;38(7):397-406. doi: https://doi.org/10.1053/j. semperi.2014.08.002.

25. Perger L, Mukhopadhyay D, Komidar L, Wiggins-Dohlvik K, Uddin MN, Beeram M. Maternal pre-eclampsia as a risk factor for necrotizing enterocolitis. The Journal of Maternal-Fetal \& Neonatal Medicine. 2016;29(13):2098-103. doi: https://doi.org /10.3109/14767058.2015.1076386. 